\title{
RNA interference-mediated silencing of the starch branching enzyme gene improves amylose content in rice
}

\author{
H. Jiang', J. Zhang ${ }^{1,2}$, J. Wang ${ }^{1}$, M. Xia ${ }^{3}$, S. Zhu' ${ }^{1}$ and B. Cheng ${ }^{1}$ \\ ${ }^{1}$ Anhui Provincial Key Laboratory of Crop Biology, \\ School of Life Science, Anhui Agricultural University, Hefei, China \\ ${ }^{2}$ Institute of Forensic Science, Ministry of Public Security, P.R. China \\ ${ }^{3}$ National Plant Gene Research Center, Beijing, China \\ Corresponding author: B. Cheng \\ E-mail: beijiucheng@ahau.edu.cn
}

Genet. Mol. Res. (2013) Ahead of Print

Received May 2, 2012

Accepted September 7, 2012

Published January 4, 2013

DOI http://dx.doi.org/10.4238/2013.January.4.19

\begin{abstract}
Amylose and amylopectin are the 2 major components of plant storage starch. The rice starch branching enzyme (RBE) plays an important role in the starch components of rice. In the present study, we selected a specific 195-bp segment from the RBE3 gene to construct hairpin DNA, which was driven by an endosperm-specific high molecular weight glutenin (Glu) promoter to regulate the biosynthesis of starch. An RNA interference (RNAi) plasmid for the RBE3 gene was constructed to form double-stranded RNA. Following Agrobacterium-mediated rice transformation (in the cultivar Zhonghua 11), 41 transgenic plants were identified using PCR and Southern blot analysis. Semi-quantitative realtime (RT)-PCR revealed that $R B E 3$ gene expression was significantly reduced in immature transgenic seeds. Transgenic rice amylose content had an average increase of $140 \%$. The highest rice amylose content was $47.61 \%$ and the growth rate increased $238 \%$ compared to the nontransgenic controls. Branching enzyme II (BEII) enzyme activity was notably reduced, and ADP-glucose pyrophosphorylase, soluble starch
\end{abstract}




\section{G.M. Guimarães-Marques et al.}

synthase, isoamylase, and pullulanase enzyme activity was markedly reduced in $\mathrm{T}_{3}$ seeds. Relative enzyme activity change explained the reduction in thousand-grain weight (TGW) in transgenic plants. The present study indicated that amylose content was negatively correlated with BEII activity, spike size, and TGW.

Key words: Rice; RBE3 gene; RNA interference; Amylose content

\section{INTRODUCTION}

Starch is the main stored carbohydrate in plants and is the primary component of food crops (Kuipers et al., 1994). Amylose and amylopectin are the 2 components of starch. Amylose is essentially a linear molecule in which glucose residues are joined via an $\alpha-(1,4)$ glucosidic bond. Amylopectin consists of highly branched glucan chains, including chains of roughly $20 \alpha-(1,4)$-linked glucose residues joined by $\alpha-(1,6)$ linkages to other branches (Mukerjea and Robyt, 2010). The proportion of amylose to amylopectin is a key factor in determining starch quality. Low amylose levels and high amylopectin levels are largely applied in food production; high amylose levels and low amylopectin levels are widely applied in industries (Liebl et al., 1997; Desai, 2007). Therefore, research aimed at regulating starch biosynthesis and improving starch quality is imperative. Developments in molecular biology have resulted in various starch biosynthesis management methods, ranging from genetic engineering to conventional breeding (Hirose and Terao, 2004; Song et al., 2004; Leterrier et al., 2008).

Starch is manufactured via the activity of starch synthesizing enzymes during a long developmental period (Fontaine et al., 1993). Starch biosynthesis involves the activities of 4 enzymes, that is, ADP-glucose pyrophosphorylase (ADPGPPase), starch synthase (SS), starch branching enzyme (SBE), and starch debranching enzyme (DBE; Ball et al., 1996); these enzymes catalyze the shape of ADP-glucose, prolong glucan chains, form branching chains, and mediate starch degradation, respectively. Previous research has demonstrated that amylose is directly catalyzed by granule-bound starch synthase (GBSS or WAXY). Furthermore, amylopectin is synthesized by the production of a joint reaction between SBE, soluble starch synthase (SSS), and DBE; therefore, rice starching branching enzyme 3 (RBE3) is considered critical in amylopectin synthesis. Previous studies indicate that the strategy for improving starch quality should focus on regulating levels of specific enzymes or on harmonizing key enzymes (Hirose and Terao, 2004; Chen et al., 2006; Zhang et al., 2007).

Previous research has shown that RNA interference (RNAi) an approach that effectively controls gene expression. To date, RNAi has been extensively employed to inhibit gene expression in plants, epiphytes, nematodes, protozoa, hexapods, and mammals (Mao et al., 2007; Jain et al., 2008a; Kim et al., 2009; McGinnis, 2009). In the present study, we constructed an $R B E 3$ gene siRNA expression plasmid for efficient regulation of rice starch, which contained an $R B E 3$ gene segment inverted repeat structure. The rice was transformed with an RNAi vector, resulting in $R B E 3$ gene expression degradation of the endogenous homologous gene, thereby obtaining an $R B E 3$-deficient mutation. These results provide basic data for $R B E 3$ function as related to starch structure and quality, and its regulation network fir crop seed characteristics. 
Silencing of $R B E 3$ gene improves amylose content in rice

\section{MATERIAL AND METHODS}

\section{PCR amplification of the RBE3 gene segment}

Rice (Oryza sativa sp japonica) variety Zhonghua 11 young leaves and immature seeds were collected at the seedling and filling stage for DNA and RNA isolation. The nucleotide sequence of the $R B E 3$ gene was verified according to the principles of siRNA design. The segment was located within 66,197 and 66,391 bp of the rice genome sequence, for a total of 195 bp. The sense primer RBE3i-F 5'-GCGGATCCGGAAGTAGCGATTAACGTGTT-3' and antisense primer RBE3i-R 5'-GCGTCGACTAGCTTTACCTTTGCCCCTT-3' were used. A $50-\mu \mathrm{L}$ reaction comprising the following was used: $1 \mu \mathrm{L} 10 \mathrm{mM}$ dNTP, $1 \mu \mathrm{L} 1 \mu \mathrm{g} / \mu \mathrm{L}$ DNA template, $3 \mu \mathrm{L} 10 \mu \mathrm{M}$ primers, $5 \mu \mathrm{L} 10 \mathrm{X}$ pfu buffer, and $1 \mu \mathrm{L} 5 \mathrm{U} / \mu \mathrm{L}$ pfu DNA polymerase, followed by addition of MilliQ $\mathrm{H}_{2} \mathrm{O}$ to reach the final volume. PCR amplification conditions were as follows: initial denaturation at $95^{\circ} \mathrm{C}$ for $5 \mathrm{~min} ; 35$ cycles of denaturation at $95^{\circ} \mathrm{C}$ for $1 \mathrm{~min}$, annealing at $56^{\circ} \mathrm{C}$ for $45 \mathrm{~s}$, extension at $72^{\circ} \mathrm{C}$ for $30 \mathrm{~s}$; followed by final extension at $72^{\circ} \mathrm{C}$ for $8 \mathrm{~min}$.

\section{Construction of siRNA expression plasmid and transformation}

The constructed siRNA expression plasmid included a 195-bp inverted repeat spliced by a gibberellin (GA)20-oxidase intron 1 fragment driven by an endosperm-specific, high molecular weight glutenin (Glu) promoter. The gene expressing the siRNA of the RBE3 gene was transferred into the pCAMBIO1301 vector, which contained the kanamycin gene as a selection marker. This plasmid was designated pRBEi.

In the T-DNA of p1300 (RBE3i), the inverted repeat was spliced by GA20-oxidase intron 1 and driven by an endosperm-specific, high molecular weight glutenin Glu-1D-1 gene promoter, using the bar gene as the selectable marker. The binary vector was transferred into Agrobacterium strain AGL0. The rice transformation system was utilized according to methods described elsewhere (Hiei et al., 1994; Yookongkaew et al., 2007).

\section{Molecular characterization of transgenic plants}

Genomic DNA of $\mathrm{T}_{0}$ transgenic plants was extracted from young leaves using the cetyltrimethylammonium bromide (CTAB) method. The primers used were as follows: specific primers 5'-ATGAGCCCAGAAGACG-3' and 5'-TCAGATCTCGGTGACGG-3' were used for amplification of the bar gene. PCR amplification conditions were as follows: initial denaturation at $95^{\circ} \mathrm{C}$ for $5 \mathrm{~min}$; followed by 35 cycles of denaturation at $95^{\circ} \mathrm{C}$ for $30 \mathrm{~s}$, annealing at $60^{\circ} \mathrm{C}$ for $30 \mathrm{~s}$, extension at $72^{\circ} \mathrm{C}$ for $30 \mathrm{~s}$; followed by a final extension at $72^{\circ} \mathrm{C}$ for $8 \mathrm{~min}$. Transgenic plants were positively identified by PCR and were further characterized using Southern blot analysis. The bar gene (550 bp) was digested from the pCAMBIA1300 plasmid and labeled with digoxigenin (DIG) as a Southern blot probe. A DIG High Prime DNA Labeling and Detection Starter Kit (Roche, Mannheim, Germany) was used for Southern blotting.

To examine expression of the $R B E 3$ gene by semi-quantitative real-time (RT)-PCR, total RNA from untransformed and transgenic plants was isolated from immature seeds (AP). The specific primers actin (Act)-F, Act-R, RBE3-F, and RBE3-R were synthesized according 
G.M. Guimarães-Marques et al.

to the rice actin and $R B E 3$ genes. A 1st-Strand cDNA Synthesis Kit (Shenergy Biocolor Company, Shanghai, China) was used for reverse transcription experiments. The actin gene was used as the internal control. PCR amplification conditions were as follows: initial denaturation at $95^{\circ} \mathrm{C}$ for $2 \mathrm{~min}$; followed by 25 cycles of denaturation at $95^{\circ} \mathrm{C}$ for $30 \mathrm{~s}$, annealing at $60^{\circ} \mathrm{C}$ for $30 \mathrm{~s}$, extension at $72^{\circ} \mathrm{C}$ for $30 \mathrm{~s}$; followed by a final extension at $72^{\circ} \mathrm{C}$ for $8 \mathrm{~min}$. The primer sequences were as follows: Act-F: 5'-CCCTTGTGTGTGACAATGGAACT-3', Act-R: 5'-GACACGGAGCTCGTTGTAGAAGG-3', RBE3-F: 5'-ATGAGTTCGGACATCCTGAAT GG-3', RBE3-R: 5'-CATTCCGCTGGAGCATAGACAAC-3'.

\section{Amylose content measurement}

The $T_{1}$ seeds were cut with clean blades, and embryos were cultured in Murashige and Skoog (MS) medium. Seedlings were cultivated in plastic plates to reproduce $T_{2}$ seeds. Homozygotes were selected by continued PPT addition. Endosperm was selected to measure amylase content by iodine colorimetry in homozygote plants.

\section{Enzyme activity and agronomic characteristic analysis of transgenic lines}

Following 15 days of flowering, rice grains were randomly chosen in each transgenic line for endosperm analysis. Branch enzyme II (BEII) enzyme activity was analyzed according to a previously described method (Mutisya et al., 2009). Ten plants were randomly chosen from each $\mathrm{T}_{3}$ generation homozygote to analyze plant height, effective number of grains per spike, thousand-grain weight (TGW), and amylose content.

Further TGW reduction was assessed in the $\mathrm{T}_{3}$ generation of $\mathrm{C} 18, \mathrm{C} 16$, and Zhonghua 11 transgenic lines to detect starch synthesis activity relative to the following enzymes: ADPGPPase enzyme activity was analyzed according to the method described by Siedlecka et al. (2003), SSS and GBSS were evaluated according to the method of Shimonaga et al. (2007), and isoamylase and pullulanase activity was assessed by the method described by Kubo et al. (1999).

\section{RESULTS}

\section{Construction of expression vector and molecular identification of transgenic rice}

The SiRNA expression vector T-DNA structure is shown in Figure 1. The PCR results indicated that 41 of 64 individual plants were positive transgenic plants, and the positive ratio was $64.6 \%$ in all plants. All $\mathrm{T}_{0}$ transgenic lines were analyzed by Southern blot probed with the bar gene. In all 6 transgenic lines, 1 single-copy transgenic event was universal, while tandem insertion of multiple copies did not occur in transgenic rice (Figure 2). Untransformed plants exhibited the absence of a band, indicating that hybridization was specific to transgenic plants.

\section{Expression of the RBE3 gene and amylose content determination}

Semi-quantitative PCR was used to determine whether RBE3 mRNA expression was reduced following induction in transgenic immature rice seeds (Figure 3). The results revealed a notable reduction in $R B E 3$ gene expression in all $\mathrm{T}_{0}$ transgenic lines. This indicated that the 
constructed dsRNA effectively inhibited RBE3 gene expression. The actin gene transcripts remained at expected levels in all of the lines.

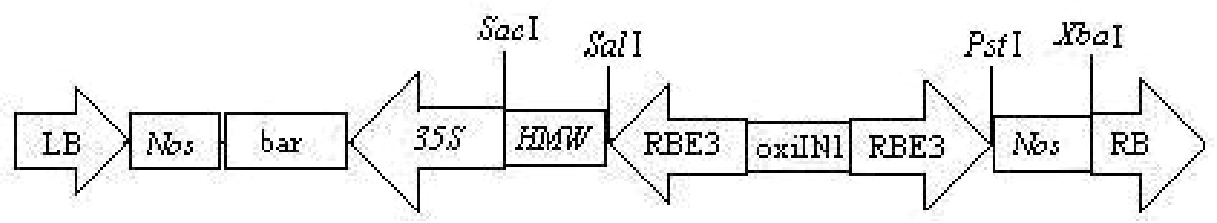

Figure 1. T-DNA structure in the pRBEi siRNA expression vector. The inverted repeat was spliced by GA20oxidase intron 1 and driven by an endosperm-specific, high molecular weight glutenin Glu promoter, using kan as the transgenic plant selectable marker.

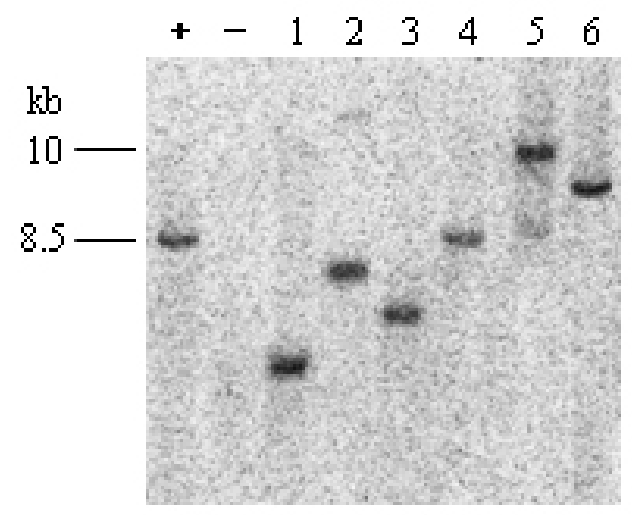

Figure 2. Transgenic plants Southern blot analysis. Lanes 1 to $6=$ Southern analysis of six transgenic plants using the bar sequence as a probe, indicating a copy insertion; lane $(-)=$ negative control, DNA from non-transformed Zhonghua11; lane $(+)=$ positive control using plasmid DNA pRBEi as a control.

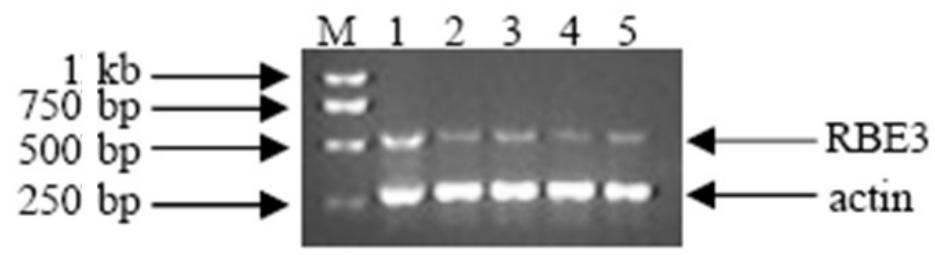

Figure 3. Semi-quantitative real-time (RT)-PCR analysis of RBE3 in $\mathrm{T}_{1}$. Lane $M=\mathrm{DNA}$ marker DL 2000; lane 1 $=$ positive control, PCR using reverse-transcribed cDNA from untransformed Zhonghua 11, an expected 510-bp RBE3 fragment was clearly evident; lanes 2 to $5=$ semi-quantitative RT-PCR analysis of four transgenic plants, the expected 510-bp RBE3 fragment was evident; internal control was a 270-bp actin fragment.

In addition, 20 of the 41 transgenic lines were randomly selected for amylose content measurements in brown rice. Corresponding amylose content was calculated using the regression equation (standard curve and regression equation are not listed). The results showed that the difference between transformed and untransformed seed amylose content was significant 
G.M. Guimarães-Marques et al.

$(\mathrm{P}<0.01$; Table 1). The performance of amylose content in all 20 transgenic lines was significantly increased by an average of $140 \%$. Amylose content increased from $14.09 \%$ in untransformed rice to $33.86 \%$ in transformed rice. Amylose content in the C18 line reached $47.61 \%$, indicating a $238 \%$ increase.

Table 1. Amylose content from different lines of $\mathrm{T}_{1}$ seeds.

\begin{tabular}{lccc}
\hline Line & Amylose content $(\%)^{\mathrm{a}}$ & Line & ${\text { Amylose content }(\%)^{\mathrm{a}}}^{\mathrm{a}}$ \\
\hline C01 & $32.27 \pm 0.19$ & $\mathrm{C} 26$ & $32.73 \pm 0.26$ \\
C02 & $39.43 \pm 0.19$ & $\mathrm{C} 27$ & $22.27 \pm 0.27$ \\
C03 & $41.93 \pm 0.23$ & $\mathrm{C} 30$ & $30.80 \pm 0.21$ \\
C07 & $34.77 \pm 0.17$ & $\mathrm{C} 32$ & $31.70 \pm 0.24$ \\
C10 & $24.43 \pm 0.22$ & $\mathrm{C} 33$ & $33.86 \pm 0.10$ \\
C12 & $37.05 \pm 0.17$ & $\mathrm{C} 38$ & $30.00 \pm 0.23$ \\
C16 & $31.82 \pm 0.19$ & $\mathrm{C} 39$ & $28.18 \pm 0.07$ \\
C18 & $47.61 \pm 0.29$ & $\mathrm{C} 41$ & $40.00 \pm 0.13$ \\
C19 & $41.59 \pm 0.30$ & $\mathrm{C} 25$ & $35.00 \pm 0.29$ \\
C23 & $29.66 \pm 0.26$ & Zhonghua11 \\
Average of transgenic seeds & $33.86 \pm 1.36$ & $32.16 \pm 0.23$ \\
\hline
\end{tabular}

Data are reported as means \pm standard error. ${ }^{a}$ Difference between amylose content in transgenic seeds and untransformed seeds was significant $(t$-test, $\mathrm{P}<0.01)$.

\section{Seed and spike traits in $T_{1}$ transgenic rice}

Transgenic rice traits were investigated by assessing $\mathrm{T}_{1}$ seed and spike attributes (Figure 4). Transgenic and control rice exhibited different traits. Seeds of $T_{1}$ transgenic rice were whitish and friable compared to untransformed seeds. Furthermore, transgenic rice seeds were opaque and wrinkled, while untransformed seeds were full. The transgenic rice spike was thin and shriveled compared to the untransformed rice spike.
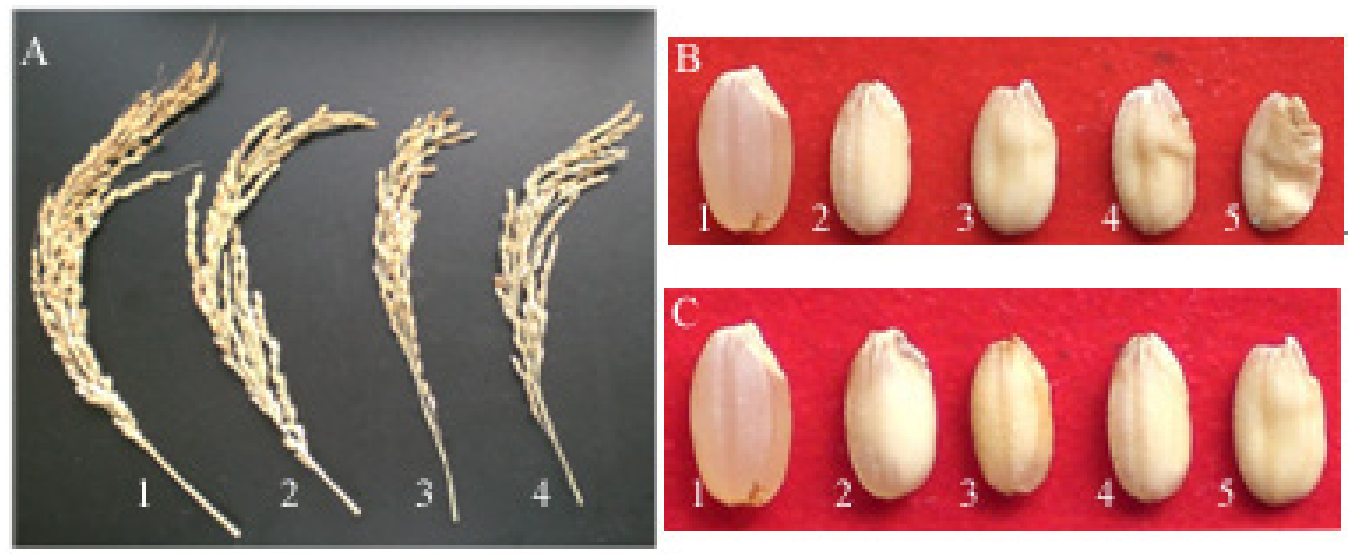

Figure 4. Transgenic traits. A. Rice spikes, control 1 depicts a robust and full untransformed rice spike; 2-4 represent thin and shriveled transgenic spikes. B. Seeds, control 1 displays an untransformed large and plump seed; 2-4 indicates small and shriveled transgenic seeds. C. Seed transparency, control 1 represents a bright seed; 2-4 displays opaque seeds. 
Silencing of $R B E 3$ gene improves amylose content in rice

\section{Agronomic traits and enzyme activities in $T_{3}$ transgenic rice}

Characterization of Zhonghua 11 and $\mathrm{T}_{3}$ transgenic rice, including amylose content, plant height, effective grain number, and TGW were investigated in detail (Table 2). Results showed that BEII enzyme activity, effective number of grains per spike, and TGW were markedly reduced in $T_{3}$ transgenic rice. In addition, transgenic rice had high amylose content relative to that of Zhonghua 11. Other key enzyme activities involved in grain starch synthesis were assessed in the $\mathrm{T}_{3}$ generation C0322 and C3911 transgenic lines. The results showed an obvious decrease in BEII enzyme activity (Table 3). The BEII enzyme activity in the C0322 transgenic line decreased by $71.1 \%$, with differences observed between lines. Furthermore, gene silencing also led to a significant reduction in ADPGPPase, SSS, and DBE activity, with a slight increase in GBSS activity.

Table 2. Trait determination in $\mathrm{T}_{3}$ transgenic rice.
\begin{tabular}{lcccc}
\hline Lines & Proportion (\%) amylose content & Plant height (cm) & Effective grain number & Thousand-grain weight (g) \\
\hline Zhonghua 11 & $14.42 \pm 0.19$ & $92.46 \pm 0.13$ & $136.1 \pm 6.9$ & $32.84 \pm 0.31$ \\
C0221 & $33.33 \pm 0.31$ & $77.96 \pm 0.42$ & $98.4 \pm 5.8$ & $30.12 \pm 0.13$ \\
C0322 & $38.43 \pm 0.24$ & $81.21 \pm 0.48$ & $84.2 \pm 8.2$ & $28.21 \pm 0.21$ \\
C1212 & $34.15 \pm 0.91$ & $88.35 \pm 0.36$ & $93.7 \pm 8.4$ & $29.43 \pm 0.19$ \\
C3321 & $31.47 \pm 0.71$ & $86.52 \pm 0.43$ & $94.2 \pm 7.7$ & $30.45 \pm 0.33$ \\
C3931 & $30.16 \pm 0.32$ & $86.46 \pm 0.51$ & $113.3 \pm 6.7$ & $29.67 \pm 0.27$ \\
C4143 & $22.31 \pm 0.19$ & $90.28 \pm 0.21$ & $118.5 \pm 7.6$ & $30.67 \pm 0.25$ \\
\hline
\end{tabular}

Table 3. Enzyme activity (U) determination in $\mathrm{T}_{3}$ transgenic rice.

\begin{tabular}{|c|c|c|c|}
\hline Enzyme types & Zhonghua 11 & Transgenic line C0322 & Transgenic line C3931 \\
\hline BEII & $32.2 \pm 0.76$ & $9.3 \pm 0.43$ & $13.7 \pm 0.52$ \\
\hline ADPG-PPase & $125.1 \pm 3.51$ & $107.6 \pm 3.09$ & $113.3 \pm 3.31$ \\
\hline GBSS & $15.6 \pm 0.32$ & $16.5 \pm 0.71$ & $16.1 \pm 0.65$ \\
\hline SSS & $55.7 \pm 1.31$ & $47.5 \pm 1.32$ & $51.7 \pm 1.39$ \\
\hline Pullulanase & $42.5 \pm 1.12$ & $37.9 \pm 1.02$ & $39.1 \pm 1.09$ \\
\hline Isoamylase & $19.5 \pm 0.72$ & $17.2 \pm 0.71$ & $17.9 \pm 0.59$ \\
\hline
\end{tabular}

\section{DISCUSSION}

Starch is an important contributing factor to grain yield and rice quality. In recent years, cloning of starch enzyme genes has improved our understanding of starch synthesis (Jain et al., 2008b; Leterrier et al., 2008). Several cDNAs encoding SBEs have been isolated from rice, wheat, maize, and barley (Kim et al., 1998; Mutisya et al., 2003; Han et al., 2007). Four classes of enzymes catalyze starch biosynthesis, that is, AGPase, SS, BE, and DBE. For example, SS elongates glucans by adding Glc residues from ADP-Glc to the glucan nonreducing ends through $\alpha-1,4$ linkages. The $W A X Y$ gene, located on rice chromosome 6 , encodes the GBSS enzyme, which plays a key role in amylose synthesis (Chen et al., 2006). BEII proteins play a large and specific role in amylopectin synthesis.

The development of molecular biology techniques makes it possible to regulate the activity of key enzymes in starch biosynthesis and modify the content and structure of starch by genetic engineering. Many plant genetic transformation systems have been successively applied, thereby 


\section{G.M. Guimarães-Marques et al.}

improving starch quality (Ryoo et al., 2007). Excessive expression of starch synthesis related genes, including expression of endogenous genes and foreign genes, have been studied in recent years (Toyota et al., 2006). The AGPP gene glgC16 (regulation of variable configuration is not sensitive) from mutant strains of Escherichia coli 618 was used to transfer a tuber-specific expressed gene promoter into potatoes. Tuber starch content increased an average of $35 \%$ in the transgenic potatoes.

Furthermore, silencing of endogenous genes by antisense RNA or RNAi technology has also resulted in significant progress. Antisense RNA technology was employed to silence expression of the GBSS gene in potatoes, resulting in a decrease in GBSS enzyme activity, which led to a sharp decline in potato tuber amylose content (down from 70 to 100\%; Kuipers et al., 1995). Similar use of antisense RNA technology in cassava, rice, and other plants also resulted in transgenic plants with improved starch content. In this study, RNAi technology was employed to silence $R B E 3$ gene expression, and the endosperm-specific promoter Glu was selected to control its expression. SiRNA structure played a specific interference role in transgenic rice, resulting in marked improvement of transgenic rice seed amylose content, with an average increase of $140 \%$. These observations indicated that the function of $R B E 3$ is dominant to amylose content in rice endosperm. Expression of $R B E 3$ was negatively correlated with amylose content.

$\mathrm{T}_{3}$ transgenic rice was further evaluated to determine trait and amylose content differences relative to controls. Amylose content of T3 transgenic rice was stable and exhibited higher levels than that of the control. Furthermore, seeds of transgenic rice were smaller than untransformed seeds, the endosperm structure was loose, and the TGW was lower than that of untransformed seeds. This may be due to the fact that the primary rice starch component is amylopectin and that the $R B E 3$ gene plays a leading role in amylopectin formation. Therefore, a reduction in activity caused a direct decrease in TGW. The enzyme activity of the $\mathrm{T}_{3}$ generation showed that $R B E 3$ RNAi not only resulted in a decrease in $R B E 3$, but amylopectin synthesis was inhibited and amylose content significantly increased. In addition, it also led to a decrease in the activity of other key starch synthesis enzymes, i.e., ADPGPPase, SSS, and DBE were significantly reduced in transgenic seeds. Consequently, endosperm synthesis was disturbed by reduced enzyme activities. This is a viable explanation for the decline of TGW in transgenic rice. Taken together, the results of the present study suggest that the RBE3 gene plays a critical role in rice grain starch synthesis. The $R B E 3$ gene affected grain amylose content and TGW; furthermore, the $R B E 3$ gene is negatively correlated with spike size in rice. This transgenic rice is important material for amylose content production in industry.

\section{ACKNOWLEDGMENTS}

Research supported by grants from the Key Projects in the National Science \& Technology Pillar Program (\#2009BADA6B00) and the Research Fund for the Doctoral Program of Higher Education of China (\#20103418120001). We thank the members of the Key Laboratory of Crop Biology of the Anhui Province for their assistance in this study.

\section{REFERENCES}

Ball S, Guan HP, James M, Myers A, et al. (1996). From glycogen to amylopectin: a model for the biogenesis of the plant starch granule. Cell 86: 349-352.

Chen G, Wang Z, Liu QQ, Xiong F, et al. (2006). Changes in the activities of enzymes involved in starch synthesis and accumulation in caryopsis of transgenic rice with antisense Wx gene. Zhi Wu Sheng Li Yu Fen Zi Sheng Wu Xue Xue 
Silencing of $R B E 3$ gene improves amylose content in rice

Bao 32: 209-216.

Desai KG (2007). Properties of tableted high-amylose corn starch-pectin blend microparticles intended for controlled delivery of diclofenac sodium. J. Biomater. Appl. 21:217-233.

Fontaine T, D'Hulst C, Maddelein ML, Routier F, et al. (1993). Toward an understanding of the biogenesis of the starch granule. Evidence that Chlamydomonas soluble starch synthase II controls the synthesis of intermediate size glucans of amylopectin. J. Biol. Chem. 268: 16223-16230.

Han Y, Sun FJ, Rosales-Mendoza S and Korban SS (2007). Three orthologs in rice, Arabidopsis, and Populus encoding starch branching enzymes (SBEs) are different from other SBE gene families in plants. Gene 401: 123-130.

Hiei Y, Ohta S, Komari T and Kumashiro T (1994). Efficient transformation of rice (Oryza sativa L.) mediated by Agrobacterium and sequence analysis of the boundaries of the T-DNA. Plant J. 6: 271-282.

Hirose T and Terao T (2004). A comprehensive expression analysis of the starch synthase gene family in rice (Oryza sativa L.). Planta 220: 9-16.

Jain A, Mondal R, Srivastava S, Prasad R, et al. (2008a). Novel mutations in emb B gene of ethambutol resistant isolates of Mycobacterium tuberculosis: a preliminary report. Indian J. Med. Res. 128: 634-639.

Jain M, Li QB and Chourey PS (2008b). Cloning and expression analyses of sucrose non-fermenting-1-related kinase 1 (SnRK1b) gene during development of sorghum and maize endosperm and its implicated role in sugar-to-starch metabolic transition. Physiol. Plant 134: 161-173.

Kim KN, Fisher DK, Gao M and Guiltinan MJ (1998). Genomic organization and promoter activity of the maize starch branching enzyme I gene. Gene 216: 233-243.

Kim Y, Han B, Titlow W, Mays CE, et al. (2009). Utility of RNAi-mediated prnp gene silencing in neuroblastoma cells permanently infected by prions: potentials and limitations. Antiviral Res. 84: 185-193.

Kubo A, Fujita N, Harada K, Matsuda T, et al. (1999). The starch-debranching enzymes isoamylase and pullulanase are both involved in amylopectin biosynthesis in rice endosperm. Plant Physiol. 121: 399-410.

Kuipers A, Jacobsen E and Visser R (1994). Formation and Deposition of Amylose in the Potato Tuber Starch Granule Are Affected by the Reduction of Granule-Bound Starch Synthase Gene Expression. Plant Cell 6: 43-52.

Kuipers AG, Soppe WJ, Jacobsen E and Visser RG (1995). Factors affecting the inhibition by antisense RNA of granulebound starch synthase gene expression in potato. Mol. Gen. Genet. 246: 745-755.

Leterrier M, Holappa LD, Broglie KE and Beckles DM (2008). Cloning, characterisation and comparative analysis of a starch synthase IV gene in wheat: functional and evolutionary implications. BMC Plant Biol. 8: 98.

Liebl W, Stemplinger I and Ruile P (1997). Properties and gene structure of the Thermotoga maritima alpha-amylase AmyA, a putative lipoprotein of a hyperthermophilic bacterium. J. Bacteriol. 179: 941-948.

Mao YB, Cai WJ, Wang JW, Hong GJ, et al. (2007). Silencing a cotton bollworm P450 monooxygenase gene by plantmediated RNAi impairs larval tolerance of gossypol. Nat. Biotechnol. 25: 1307-1313.

McGinnis K (2009). Use of transgene-induced RNAi to regulate endogenous gene expression. Methods Mol. Biol. 526: 91-99.

Mukerjea R and Robyt JF (2010). Isolation, structure, and characterization of the putative soluble amyloses from potato, wheat, and rice starches. Carbohydr. Res. 345: 449-451.

Mutisya J, Sathish P, Sun C, Andersson L, et al. (2003). Starch branching enzymes in sorghum (Sorghum bicolor) and barley (Hordeum vulgare): comparative analyses of enzyme structure and gene expression. J. Plant Physiol. 160: 921-930.

Mutisya J, Sun C, Rosenquist S, Baguma Y, et al. (2009). Diurnal oscillation of SBE expression in sorghum endosperm. J. Plant Physiol. 166: 428-434.

Ryoo N, Yu C, Park CS, Baik MY, et al. (2007). Knockout of a starch synthase gene OsSSIIIa/Flo5 causes white-core floury endosperm in rice (Oryza sativa L.). Plant Cell Rep. 26: 1083-1095.

Shimonaga T, Fujiwara S, Kaneko M, Izumo A, et al. (2007). Variation in storage alpha-polyglucans of red algae: amylose and semi-amylopectin types in Porphyridium and glycogen type in Cyanidium. Mar. Biotechnol. 9: 192-202.

Siedlecka A, Ciereszko I, Mellerowicz E, Martz F, et al. (2003). The small subunit ADP-glucose pyrophosphorylase (ApS) promoter mediates okadaic acid-sensitive uidA expression in starch-synthesizing tissues and cells in Arabidopsis. Planta 217: 184-192.

Song JM, Li BY, You MS, Liang RQ, et al. (2004). Molecular identification of wheat granule bound starch synthase gene polymorphism. Yi Chuan Xue Bao 31: 81-86.

Toyota K, Tamura M, Ohdan T and Nakamura Y (2006). Expression profiling of starch metabolism-related plastidic translocator genes in rice. Planta 223: 248-257.

Yookongkaew N, Srivatanakul M and Narangajavana J (2007). Development of genotype-independent regeneration system for transformation of rice (Oryza sativa ssp. indica). J. Plant Res. 120: 237-245.

Zhang JJ, Hu YF, Zhou H and Huang YB (2007). Starch accumulation and activities of key enzymes involved in starch synthesis in the grains of maize inbred lines with different starch contents. Zhi Wu Sheng Li Yu Fen Zi Sheng Wu Xue Хие Вао 33: 123-130. 